\title{
Multidimensional set switching
}

\author{
SOWON HAHN \\ University of Oklahoma, Norman, Oklahoma \\ GEORGE J. ANDERSEN \\ University of California, Riverside, California \\ and \\ ARTHUR F. KRAMER \\ University of Illinois at Urbana-Champaign, Champaign, Illinois
}

\begin{abstract}
The present study examined the organization of preparatory processes that underlie set switching and, more specifically, switch costs. On each trial, subjects performed one of two perceptual judgment tasks, color or shape discrimination. Subjects also responded with one of two different response sets. The task set and/or the response set switched from one to the other after 2-6 repeated trials. Response set, task set, and double set switches were performed in both blocked and randomized conditions. Subjects performed with short (100-msec) and long (800-msec) preparatory intervals. Task and response set switches had an additive effect on reaction times (RTs) in the blocked condition. Such a pattern of results suggests a serial organization of preparatory processes when the nature of switches is predictable. However, task and response set switches had an underadditive effect on RTs in the random condition when subjects performed with a brief cue-to-target interval. This pattern of results suggests overlapping task and response set preparation. These findings are discussed in terms of strategic control of preparatory processes in set switching.
\end{abstract}

In recent years, the task switching paradigm has become an increasingly popular test bed for the examination of executive control processes involved in the coordination of multiple tasks or skills (Allport, Styles, \& Hsieh, 1994; De Jong, 2000; Gopher, 1996; Goschke, 2000; Jersild, 1927; Kramer, Hahn, \& Gopher, 1999; Mayr \& Keele, 2000; Meiran, 1996; Rogers \& Monsell, 1995). The task switching paradigm involves two important conditions or trial types: trials on which the same task is repeated (i.e., nonswitch trials), and trials on which subjects are required to switch from one task to another (i.e., switch trials). Switch costs are defined in terms of the difference between reaction time (RT) or error rate on the nonswitch (repeated) and on the switch trials. Switch costs have been interpreted in terms of the set reconfiguration operations that must occur as preparation to perform the subsequent task (Gopher, 1996; Jersild, 1927; Kramer et al., 1999; Meiran, 1996; Rogers \& Monsell, 1995; though see Allport et al., 1994, and Allport \& Wylie, 2000, for another interpretation of switch costs).

The research and preparation of this manuscript was supported by grants from the National Eye Institute (NEI EY 12437-01) and the National Institutes of Health (RO1 AG14966). We thank Veronica Dark, Katherine Arbuthnott, Ulrich Mayr, Padmanabhan Sudevan, and an anonymous reviewer for their very helpful comments on a previous draft of this manuscript. Correspondence should be sent to S. Hahn, Department of Psychology, University of Oklahoma, 455 West Lindsey Street, Norman, OK 73019-2007 (e-mail: sowon@ ou.edu).
Studies that have examined the task switching paradigm have commonly suggested that the preparatory processes underlying task switching are separate from the task processes necessary for performing the individual tasks (i.e., perceptual, decision, and motor processes). For example, Gopher, Armony, and Greenshpan (2000) reported that instructions concerning the likelihood and nature of a switch influenced switching time but not component task time (i.e., nonswitch time). Rogers and Monsell (1995) found that the time allotted to prepare for a task switch had a substantially larger influence on switch time than it did on component task time, whereas the presence of a warning cue influenced component task time but not switching time. Rubinstein, Meyer, and Evans (2001; see also Lauber, Meyer, Gmeindl, Evans, \& Kieras, 1996) reported a number of dissociations between nonswitch and switch performance across 14 different task pairs in five separate studies.

These studies are important in that they suggest a dissociation between processes that subserve the performance of the individual tasks and the processes that are involved in switching between tasks. However, another important question, not addressed by the studies reviewed thus far, is how the processes that support task switching are organized. For example, given that multiple processes are involved in task switching (Gopher et al., 2000; Rogers \& Monsell, 1995; Rubinsteinet al., 2001), such processes could be organized sequentially as discrete independent processing stages (Sternberg, 1998), or they could be performed in parallel. In the former case, sequential process- 
ing might be the result of the need for a common mechanism or controller that cannot coordinate multiple preparatory processes at the same time. In the latter case, the controller might be capable of coordinating multiple preparatory processes at the same time (Meyer \& Kieras, 1997), or there might be multiple controllers.

The literature on this issue has produced mixed results. Allport et al. (1994) had subjects switch either the type of numerical judgment (i.e., even/odd or $>/<5$ ) that they performed or the stimulus set (i.e., numerical value or group size) on which they performed the judgment or both the judgment and stimulus set. They found an underadditive interaction between judgment and stimulus type; that is, performing both switches took no longer than performing either the judgment or the stimulus switch alone. Such a pattern of data can be interpreted as suggesting that preparation to switch judgments and stimulus sets can be carried out concurrently. Kleinsorge and Heuer (1999) had subjects switch tasks (i.e., numerical vs. spatial judgments) and response mappings (i.e., compatible vs. incompatible, based on response mappings assigned to subjects) both separately and together. They observed an underadditive interaction between task and response mapping switches, suggesting, as in Allport et al.'s study, that the two preparatory operations that they examined could be performed in parallel. Finally, Gopher et al. (2000) examined the costs of switching between task sets and switching between speed/accuracy strategies. They found that costs for switching between task sets could be reduced with additional preparation time. However, increased preparation time did not help subjects switch between speed/accuracy strategies, suggesting different control operations for speed/accuracy strategy and task set switching. However, whether such operations could be performed concurrently or serially was not addressed. Thus, in summary, the literature is mixed with regard to whether multiple preparatory operations, which subserve repeatedly switching between task dimensions, are accomplished in series or in parallel.

In the present study, we further examined the organization of preparatory processes that support switching between task dimensions. We asked subjects to perform two different perceptual tasks (i.e., color and shape discrimination) with two different response sets (i.e., different response keys in different physical arrangements; see Figure 1). On any particular trial, subjects could perform the same perceptual discrimination task with the same response set (nonswitch trial), the same task with a different response set (response set switch trial), a different task with the same response set from the previous trial (task set switch trial), or a different task with a different response set (double switch trial).

We employed additive factors logic (Sternberg, 1998) to examine the organization of preparatory processes employed to switch between tasks and response sets. Evidence in support of sequential organization of task and response set preparatory processes would be provided by additivity in response time for these two factors. On the other hand, an interaction between response set and task set factors might suggest shared preparatory control.

Given our assumption that the processes underlying task and response set switches are, at least in part, preparatory in nature, we also manipulated the cue-to-target interval (CTI: 100 and $800 \mathrm{msec}$ ). We expected that switch costs, for

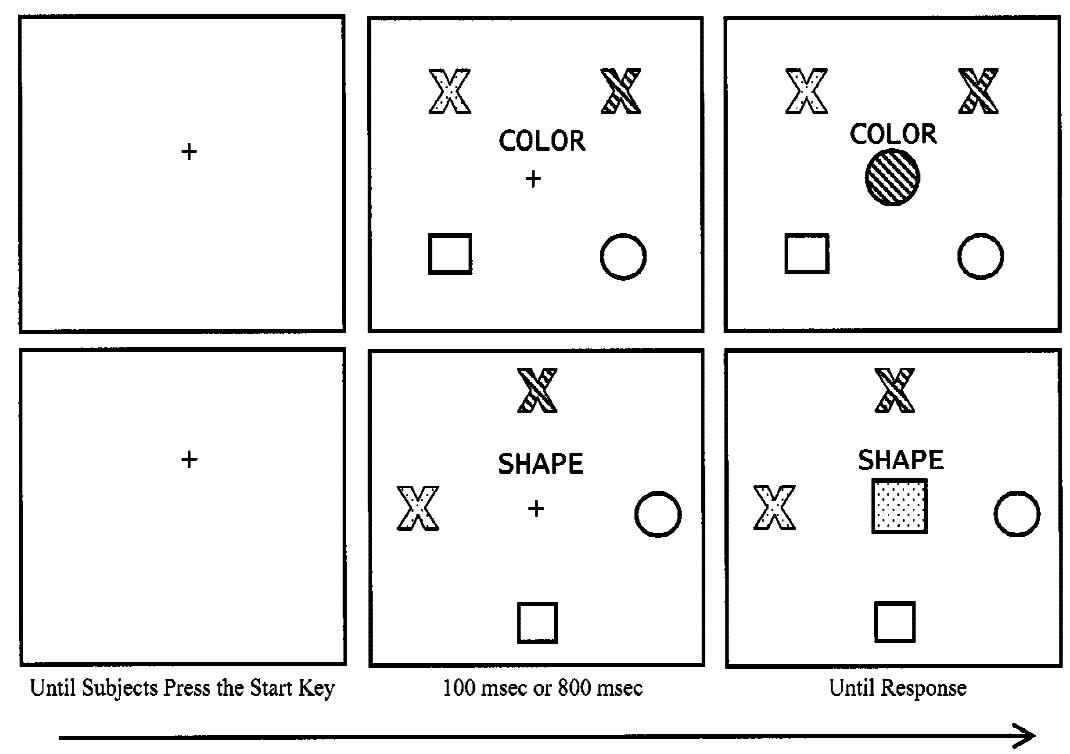

Figure 1. A schematic illustration of the trial sequence. The upper panels provide an example of the color judgment task with a square shape response key set, and the lower panels show an example of the shape judgment task with a diamond shape response key set. The dotted and lined patterns on the centrally located objects indicate the red and green colors. 
both task and response sets, would be reduced at the longer CTI (Kramer et al., 1999; Rogers \& Monsell, 1995). Furthermore, we note that the data obtained in the brief CTI condition is most relevant to the issue of how preparatory processes are organized, because it is in this condition that preparatory processes will be reflected in RT.

We also manipulated the manner in which switch and nonswitch trials were presented to subjects, either in a blocked (e.g., separate trial blocks for task set switch, response set switch, and double switch trials) or randomized fashion. This manipulation is important in that it offers the potential to provide insights into the role of subject strategies in task switching. For example, in random conditions, the fact that all tasks and response sets are relevant (i.e., subjects can be asked to switch from any combination of task and response sets to any other combination of task and response set) may encourage subjects to utilize a control structure that entails the activation of all task-response mappings (see Kleinsorge \& Heuer, 1999). Such a strategy might be expected to lead to partially concurrent processing of task and response sets, as reflected in an underadditive interaction. On the other hand, the fact that only a subset of the task-response mappings are relevant in blocked conditions may encourage subjects to deal separately with different mappings. Such a strategy might be expected to lead to serial activation of task and response set switching operations, reflected in an additive pattern of RTs for these factors.

\section{METHOD}

\section{Subjects}

Eighteen (10 female) paid subjects, 18-21 years old, participated in the experiment. All of the subjects had normal color vision tested by the Ishihara color blindness test and normal/corrected normal visual acuity tested with a Snellen chart.

\section{Apparatus}

Stimulus presentation and response collection were controlled with a CompUSA 700-Hz Pentium PC with a 19-in. Mitsubishi Precise Point $8705 \mathrm{P}$ color monitor. The timing of stimulus presentation and collection of subjects' responses were controlled with the RTX 4.2 for Windows NT from Venture Com. Subjects responded with the numeric keypad on a standard IBM keyboard.

\section{Task and Stimuli}

For each trial, subjects performed one of two tasks, color or shape discrimination. The task to be performed on a particular trial was indicated with a written cue, SHAPE or COLOR, presented above the target stimulus (see Figure 1). The imperative stimulus $(2 \times 2 \mathrm{~cm}$ in height and width) was one of four objects - a red circle, green circle, red square, or green square- that was presented in the center of the computer display. For each trial, subjects responded by pressing one of the four possible keys on the numeric keypad. Two different stimulus-response mapping sets were used: one response set included the "1," "3", "7," and "9" keys of the numeric keypad, which are arranged as a square shape, and the other response set included the " 2 ," " 4 ," " 6 ," and " 8 " keys of the numeric keypad, which are arranged as a diamond shape. The keys used in two response sets did not overlap, so there was no conflict between the keys in the two response sets. For each response set, two keys were used for responding on the color task, and two keys for responding on the shape task. The task-response mappings were counterbalanced across subjects.
The response set on a particular trial was indicated by the arrangement of symbolic cues presented on the display, including four objects representing each response (a red letter X, a green letter X, a gray circle, and a gray square). The response cue objects were arranged with the same spatial configuration as that of the response key set, in a square or diamond shape as indicated in Figure 1. To aid the subjects in discerning the task set and response set assignments, the cues for these sets remained on the screen throughout the trial.

\section{Procedure}

Subjects sat approximately $60 \mathrm{~cm}$ from the screen. On each trial, the display started with a fixation cross $(1 \times 1 \mathrm{~cm})$ presented in the center of the screen. When subjects were ready, they started the trial by pressing the " 5 " key on the numeric keypad. After subjects pressed the key, a task cue and a response set cue appeared simultaneously on the screen. After 100 or $800 \mathrm{msec}$ (short or long CTI), a target stimulus replaced the fixation cross in the center of the display. The task cue, the S-R mapping cue, and the centrally located target stimulus remained on the screen until subjects responded. Following the subjects' response, a fixation cross for the next trial appeared on the screen and remained until subjects started the trial by pressing the " 5 " key on the numeric keypad. Thus, each trial was self-paced. A trial sequence is illustrated in Figure 1.

The task switch and response set switch occurred quasi-randomly, so that for each trial, subjects could either repeat or switch from the previous task and response sets. The switch for task and response set occurred after two to six repeated trials. Thus, each of the following runs occurred with equal probability; S-R, S-R-R, S-R-R-R, S-R-R-R-R, and S-R-R-R-R-R (with S = switch, R = repeated). The task switch and the response set switch could occur either simultaneously or separately.

\section{Design}

Each subject participated in two experimental sessions. For one session, the three different switch types-a task switch (i.e., color or shape task), response set switch (i.e., diamond shaped response set or squared shaped response set), and a double switch-were blocked. For the other session, the three different switch types (i.e., task set switch, response set switch, double switch) were randomized within blocks. The order of the blocked and mixed sessions was counterbalanced over subjects. The short (100-msec) and long (800-msec) CTIs were blocked within each session.

The blocked switch session included six sub-blocks, two task set switch blocks, two response set switch blocks, and two double switch blocks. Each of the different types of switch blocks included one short and one long CTI block. Each block consisted of 240 trials, including 60 switch trials and 180 nonswitch trials. ${ }^{1}$ The order of block types and the order of the specific task set and response set pairings was counterbalanced over subjects. However, it is important to note that the specific response keys (within the rectangle and diamond response key arrangements) to task dimensions arrangements were constant for each subject (e.g., the mapping of the "8" and " 6 " keys to the red and green stimuli, respectively).

The randomized switch session included six blocks-three short and three long CTI blocks-and three different types of switches were randomized within each block. Before each session, subjects participated in a short practice block of 80 trials.

\section{RESULTS}

The RT data were submitted to a four-way repeated measures analysis of variance with block type (blocked, random), CTI (100 msec, $800 \mathrm{msec}$ ), task set switch (task nonswitch trials, task switch trials), and response set switch (response set nonswitch trials, response set switch trials) as within-subjects variables. ${ }^{2}$ The RT data are presented in 

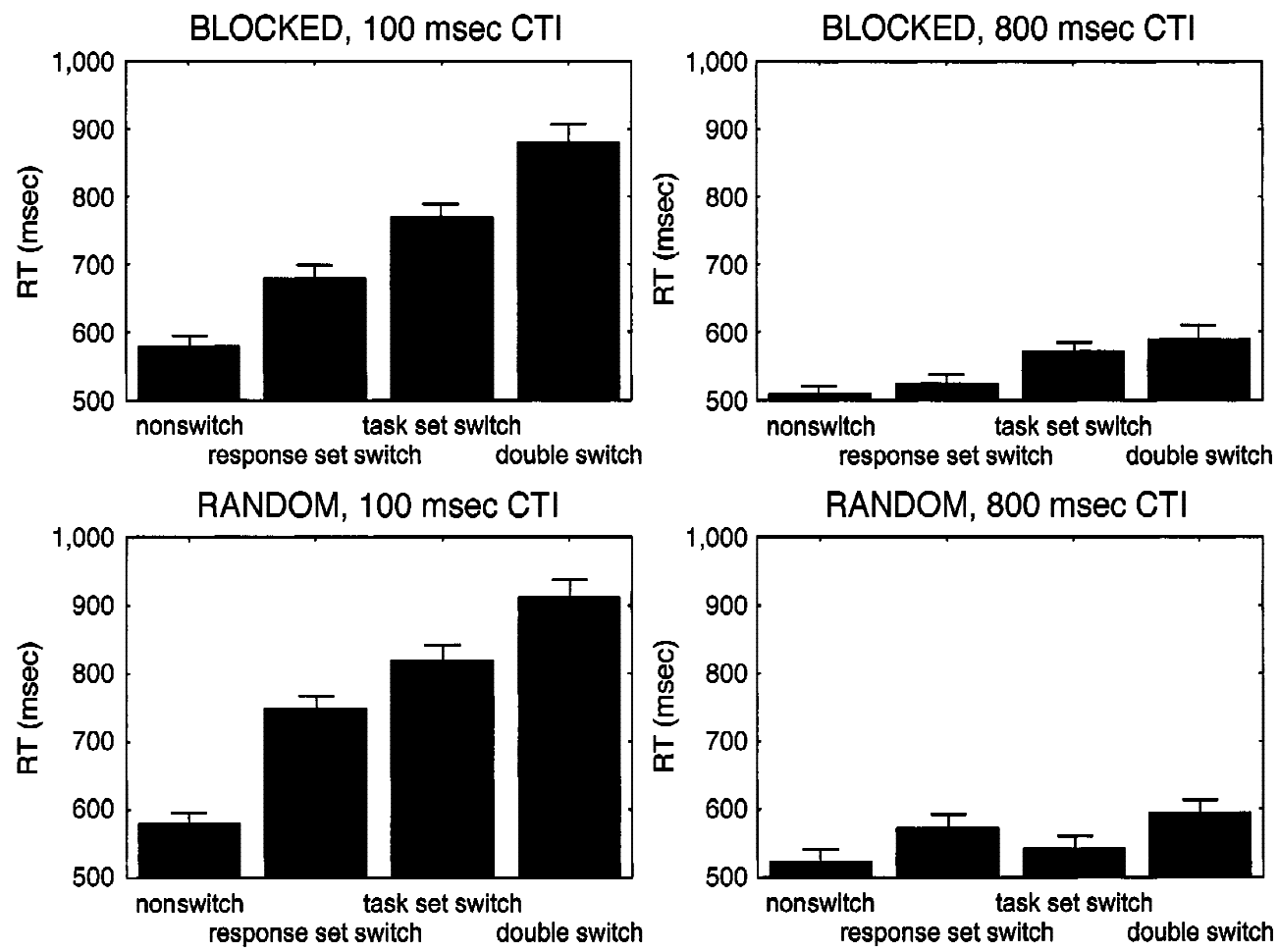

Figure 2. Mean reaction times (RTs) with standard error bars for each of the conditions in the study.

Figure 2. Main effects were obtained for the CTI $[F(1,17)=$ $\left.175.6, M S_{\mathrm{e}}=23,533, p<.01\right]$, task set switch $[F(1,17)=$ $\left.129.7, M S_{\mathrm{e}}=15,211, p<.01\right]$, and response set switch $\left[F(1,17)=41.1, M S_{\mathrm{e}}=8,027, p<.01\right]$ variables. $\mathrm{RTs}$ were faster for the longer than for the shorter preparation interval. RTs were faster when subjects repeatedly performed the same task than when they switched between the color and shape judgment tasks. Finally, RTs were also faster when subjects repeatedly used the same response set than when they switched between the diamond and square response sets.

The main effects were qualified by a number of significant interactions. Significant two-way interactions were obtained for block $\times$ CTI $\left[F(1,17)=5.2, M S_{\mathrm{e}}=2,903\right.$, $p<.01]$, CTI $\times$ task switch $\left[F(1,17)=158.7, M S_{\mathrm{e}}=\right.$ $2,795, p<.01]$, and CTI $\times$ response switch $[F(1,17)=$ $\left.37.8, M S_{\mathrm{e}}=3,356, p<.01\right]$. None of the other two- or three-way interactions was significant. As can be seen in Figure 2, switch costs were substantially larger when subjects had $100 \mathrm{msec}$ to prepare for the subsequent task or response set (199-msec and 118-msec switch costs for task and response set switches, respectively) than when they had $800 \mathrm{msec}$ to prepare for the next task or response set (41-msec and 34-msec switch costs for task and response set switches, respectively). These results are consistent with those of previous studies in which subjects were found to capitalize on longer preparation times in order to reduce switch costs (Gopher et al., 2000; Kramer et al., 1999; Rogers \& Monsell, 1995). Subjects also showed larger performance costs for the random than for the blocked conditions when given only $100 \mathrm{msec}$ to prepare for the next task (39 msec slower for the random than for the blocked conditions) but essentially equivalent performance when given $800 \mathrm{msec}$ to prepare $(9 \mathrm{msec}$ slower for the random than for the blocked conditions).

Finally, and most importantly, we obtained a significant four-way interaction for RT $\left[F(1,17)=5.3, M S_{\mathrm{e}}=1,651\right.$, $p<.05]$. Inspection of Figure 2 suggests that response set $\times$ task set effects are additive for the blocked 100msec and 800-msec CTIs and for the random 800-msec CTI condition. On the other hand, the task set $\times$ response set appears to be underadditive for the random $100-\mathrm{msec}$ CTI condition. These observations were consistent with planned comparisons. The task set $\times$ response set interaction was significant for only the random 100 -msec CTI condition $\left[F(1,17)=8.9, M S_{\mathrm{e}}=2,920, p<.01\right]$. In this case an underadditiveinteraction was obtained for the task set $\times$ response set contrast such that the response set switch minus nonswitch effect was $169 \mathrm{msec}$ when the task set was repeated and $93 \mathrm{msec}$ when subjects also switched task sets. The $F$ ratios for the three nonsignificant task $\times$ response set contrasts were $F(1,17)=0.20$, $M S_{\mathrm{e}}=2,662, p>.65$, for 100 -msec CTI blocked; $F(1,17)=$ $0.02, M S_{\mathrm{e}}=1,659, p>.88$, for the 800-msec CTI blocked; and $F(1,17)=0.04, M S_{\mathrm{e}}=1,359, p>.83$, for the 800 msec CTI random condition.

The error rate data were also submitted to a four-way repeated measures analysis of variance with block type 
(blocked, random), CTI (100 msec, $800 \mathrm{msec})$, task set switch (task nonswitch trials, task switch trials), and response set switch (response set nonswitch trials, response set switch trials) as within-subjects variables. The error rate data are presented in Table 1. Response set errors could occur only for the relevant response set, because the other response set keys were inactivated (i.e., if the square response set was relevant, the diamond response set was inactivated). Main effects were obtained for the CTI $[F(1,17)=$ $\left.5.6, M S_{\mathrm{e}}=0.0012, p<.05\right]$ and task set switch $[F(1,17)=$ $\left.82.9, M S_{\mathrm{e}}=0.0015, p<.01\right]$ variables. Error rates were smaller for the longer than for the shorter preparation interval. Error rates were also smaller when subjects repeatedly performed the same task than when they switched between the color and shape judgment tasks. These effects are consistent with the RT effects reported above.

A significant two-way interaction was also obtained for the CTI $\times$ task switch variables $\left[F(1,17)=5.8, M S_{\mathrm{e}}=\right.$ $0.0006, p<.05]$. There was a larger cost to task switching in error rates for short than for long preparation intervals $(4.2 \%$ and $2.7 \%$ for the 100 -msec and $800-\mathrm{msec}$ CTIs, respectively). None of the other two- or three-way interactions was significant.

\section{DISCUSSION}

The main question addressed in the present study was how preparatory processes are organized when subjects are required to switch among different task and response dimensions. We therefore manipulated the task and response sets with which subjects performed the experimental tasks. We also varied the CTI in an effort to verify that the operations necessary for switching task dimensions could be carried out, on the basis of instructions, in advance of the imperative stimuli. Finally, the manipulation of trial blocking enabled us to examine potential strategic effects in the organization of task and response set switching operations.

We found that switch costs, for both task and response sets, were reduced with longer CTIs. Given our assumption that the processes underlying set switches are preparatory in nature, this finding was expected. In addition, because it is in the brief CTI condition that preparatory processes will be reflected in RT, we focus our discussion on this condition.

We found that the influence of the task set and response set switches on RT varied as a function of whether trial types were blocked or randomized. Under blocked conditions, we observed an additive effect of task set and response set variables on RT. However, under random conditions, task and response set had an underadditive effect on RT. This pattern was found for both long and short CTIs under blocked conditions, and for the short CTI under random conditions. These results are interesting from a number of perspectives. First, although other researchers (Allport et al., 1994; Kleinsorge \& Heuer, 1999) have examined combinations of components of task set reconfiguration in task switching, they have done so in either blocked or random conditions, rather than contrasting performance under both conditions. As the present data illustrate, the influence of different set variables on RT may vary under blocked and random conditions, suggesting that subjects' strategies may play an important role in the organization of preparatory processing in task switching.

Second, the use of both blocked and random conditions with the same subjects and task parameters enabled us to examine the potential impact of subjects' strategies on task switching. We suggested, in agreement with Kleinsorge and Heuer's (1999) control structure model of task switching, that in random conditions the fact that all tasks and response sets are relevant (i.e., subjects can be asked to switch from any combination of task and response sets to any other combination of task and response set) may encourage subjects to utilize a control structure that entails the activation of all task-response mappings (see Kleinsorge \& Heuer, 1999). Such a strategy might be expected to lead to partially concurrent processing of task and response sets, reflected in an underadditive interaction, particularly for the short CTI. On the other hand, the fact that only a subset of the task-response mappings is relevant in blocked conditions may encourage subjects to deal separately with different mappings. Such a strategy might be expected to lead to serial activation of task and response set switching operations, reflected in an additive pattern of RTs for these variables. The RT data were con-

Table 1

Mean Error Rates for Task (Color and Shape) and Response Set (Diamond and Square Arrangement) for Both Blocked and Randomized Conditions at the Short (100-msec) and Long (800-msec) Cue to Target Intervals

\begin{tabular}{|c|c|c|c|c|c|c|c|c|}
\hline \multirow[b]{4}{*}{ Condition } & \multicolumn{8}{|c|}{ Switch Condition } \\
\hline & \multicolumn{4}{|c|}{ Repeated Task } & \multicolumn{4}{|c|}{ Switched Task } \\
\hline & \multicolumn{2}{|c|}{ Response Set (R) } & \multicolumn{2}{|c|}{ Response Set (S) } & \multicolumn{2}{|c|}{ Response Set (R) } & \multicolumn{2}{|c|}{ Response Set (S) } \\
\hline & $M$ & $S E$ & $M$ & $S E$ & $M$ & $S E$ & $M$ & $S E$ \\
\hline Blocked 100 msec & 3.5 & 0.5 & 1.9 & 0.4 & 7.7 & 1.1 & 7.8 & 1.0 \\
\hline Blocked 800 msec & 2.2 & 0.4 & 2.4 & 0.6 & 5.8 & 0.8 & 4.6 & 0.8 \\
\hline Random $100 \mathrm{msec}$ & 2.8 & 0.3 & 3.1 & 0.8 & 6.6 & 0.6 & 5.5 & 0.9 \\
\hline Random $800 \mathrm{msec}$ & 1.9 & 0.4 & 3.2 & 0.8 & 5.6 & 1.0 & 4.6 & 1.0 \\
\hline
\end{tabular}

Note-R indicates that the response set repeats; $\mathrm{S}$ indicates that the response set switches. 
sistent with these predictions. With short preparatory intervals, an underadditive interaction was obtained for the task and response set variables. However, an additive RT pattern was obtained in the blocked conditions.

These data may also be interpreted in the context of Mayr and Kliegl's (2000) long-term memory (LTM) retrieval model of task switching. ${ }^{3}$ Mayr and Kliegl have argued that switch costs, by and large, reflect the time required by subjects to retrieve the appropriate task rules from LTM. This conclusion is consistent with their finding that switch costs are larger when the switches involve high retrieval demands than when they involve low retrieval demands. In the present experiment, one might have expected subjects to have particular difficulties in the random condition when a single dimension was switched (i.e., either the task or response set), because under these conditions the binding between the two dimensions needed to be changed. Evidence in support of this speculation is provided by the fact that underadditivity of the random $100-\mathrm{msec}$ CTI condition was found through increased single-dimension switch costs-in comparison with the blocked 100-msec CTI condition-instead of decreased double-dimensionswitch costs (see Figure 2). No such effects would be expected in the blocked conditions, because only a subset of dimensions is relevant in any particular trial block.

The present data do not permit us to distinguish between Kleinsorge and Heuer's (1999) control structure and Mayr and Kliegl's (2000) LTM retrieval account of switch costs. Indeed, these explanations are not necessarily mutually exclusive, because control structures are likely to be constructed and maintained in LTM. Future studies will be needed to examine this issue further.

Kleinsorge and Heuer (1999) used a randomized design and found that judgment type and response compatibility had an underadditive effect on RT, a pattern of data similar to our finding of underadditivity with task and response set switches in the random condition with short CTI. However, Allport et al. (1994) employed a blocked design and found that numerical operation and stimulus dimension variables had an underadditive effect on RT. This is quite different from our finding, in blocked conditions, of an additive RT pattern for task and response set switching. What might be the source of the different patterns of effects for blocked conditions in the present study and in Allport et al.? One possibility concerns the way in which the relevant dimensions were cued in the two studies. In Allport et al., subjects needed to keep track of which dimension was relevant on any particular trial, whereas in the present study (see Figure 1), the task set and response set were explicitly cued on each trial. Thus, it is conceivable that internal and external cuing might have led to different preparatory strategies.

Clearly, additional studies will be required to examine the generalizability of the effects observed in the present study. It would be prudent if such studies included both random and blocked conditions, in order to enable the ex- amination of strategic effects on task switching. Such studies might also examine the influence of different task dimensions on the ability of subjects to strategically adjust the manner in which they organize different preparatory processes in the service of task switching. In summary, the results of the present study clearly suggest that multiple switching operations can be implemented in either a sequential or a concurrent fashion. Future studies will be necessary to examine the boundary conditions on these effects.

\section{REFERENCES}

Allport, A., Styles, E. A., \& Hsieh, S. (1994). Shifting intentional set: Exploring the dynamics of tasks. In C. Umiltà \& M. Moscovitch (Eds.), Attention and performance XV (pp. 421-452). Hillsdale, NJ: Erlbaum.

Allport, A., \& Wy lie, G. (2000). Task switching, stimulus-response bindings, and negative priming. In S. Monsell \& J. Driver (Eds.), Attention and performance XVIII (pp. 35-70). Cambridge, MA: MIT Press.

DE Jong, R. (2000). An intention-activation account of residual switch costs. In S. Monsell \& J. Driver (Eds.), Attention and performance XVIII: Control of cognitive processes ( pp. 357-376). Cambridge, MA: MIT Press.

GoPHER, D. (1996). Attentional control: Explorations of the work of an executive controller. Cognitive Brain Research, 5, 23-38.

Gopher, D., Armony, L., \& Greenshran, Y. (2000). Switching tasks and attention policies. Journal of Experimental Psychology: General, 129, 308-339.

Goschke, T. (2000). Intentional reconfiguration and involuntary persistence in task set switching. In S. Monsell \& J. Driver (Eds.), Attention and performance XVIII (pp. 331-356). Cambridge, MA: MIT Press.

Jersild, A. T. (1927). Mental set and shift. Archives of Psychology, 14(No. 89)

Kleinsorge, T., \& Heuer, H. (1999). Hierarchical switching in a multidimensional task space. Psychological Research, 62, 300-312.

Kramer, A. F., Hahn, S., \& Gopher, D. (1999). Task coordination and aging: Explorations of executive control processes in the task switching paradigm. Acta Psychologica, 101, 339-378.

Lauber, E. J., Meyer, D. E., Gmeindl, L., Evans, J., \& Kieras, D. E. (1996, November). The executive processes involved in task switching. Paper presented at the 37th Annual Meeting of the Psychonomic Society, Chicago.

Mayr, U., \& KeEle, S. W. (2000). Changing the internal constraints on action: The role of backward inhibition. Journal of Experimental Psychology: General, 129, 4-26.

MAYR, U., \& KLIEGL, R. (2000). Task set switching and long term memory retrieval. Journal of Experimental Psychology: Learning, Memory, \& Cognition, 26, 1124-1140.

Meiran, N. (1996). Reconfiguration of processing mode prior to task performance. Journal of Experimental Psychology: Learning, Memory, \& Cognition, 22, 1423-1442.

Meyer, D. E., \& Kieras, D. E. (1997). A computational theory of executive control processes and multiple task performance: I. Basic mechanisms. Psychological Review, 104, 3-65.

Rogers, R. D., \& Monsell, S. (1995). Costs of a predictable switch between simple cognitive tasks. Journal of Experimental Psychology: General, 124, 207-231.

Rubinstein, J., Meyer, D., \& Evans, J. (2001). Executive control of cognitive processes in task switching. Journal of Experimental Psychology: Human Perception \& Performance, 27, 763-797.

Sternberg, S. (1998). Discovering mental processing stages: The method of additive factors. In D. Scarborough \& S. Sternberg (Eds.), Methods, models and conceptual issues: An invitation to cognitive science (Vol. 4, pp. 703-863). Cambridge, MA: MIT Press. 


\section{NOTES}

1. To ensure that subjects were exposed to all pairings of task and response set arrangements in the blocks, a switch was made mid-block (i.e., after the first 120 trials). For example, in the task switch blocks, subjects who started out with the diamond response key arrangement switched to the rectangle response key arrangement mid-block. This switch was explicitly indicated to subjects with instructions on the computer screen, and the trial immediately after the mid-block switch of the response set was discarded. The same procedure was used in the response set switch blocks as well as in the double switch blocks to ensure that subjects were exposed to all combinations of task set and response set arrangements. To ensure that this change did not influence the pattern of
RT, before and after the dimensional change, we performed an analysis of variance with first/second half of block as a variable. The effect of this variable was not significant, nor did this variable interact with other experimental variables.

2 . We collapsed across all of the nonswitch trials (from different run lengths) following a switch because the RTs did not significantly differ across these trials.

3. We would like to thank Ulrich Mayr for suggesting this possibility.

(Manuscript received April 18, 2000;

revision accepted for publication March 20, 2002.) 